\title{
MECHANICAL INSTABILITY OF SNOWCOVER WITH SATURATED LAYER
}

\author{
by
}

\section{YASUAKI NOHGUCHI}

Institute of Snow and Ice Studies,

NRCDP, Science and Technology Agency, Suyoshi-cho, Nagaoka, Japan 940

\section{ABSTRACT}

If rain falls on a new snowcover, a water saturated layer is of ten clearly formed in it. A snowcover with a saturated layer is sometimes unstable; the snowcover is folded and dimples appear on the snow surface. The mechanism of this instability is discussed theoretically. As a result, we find that a snowcover with a saturated layer can be unstable owing to lateral movement of water along a saturated layer and elastic deformation of the snow layer directly under it. Then the wavelength of the instability depends on the snow density; this result is consistent with field observations.

\section{INTRODUCTION}

The dimple pattern (Figure 1) due to rainfall or snow melting had been observed by many researchers; Seligman (1936), Gerdel (1954), Wakahama (1963), Wankiewicz and de Vries (1978), and Nohguchi (1984). Whenever dimples begin

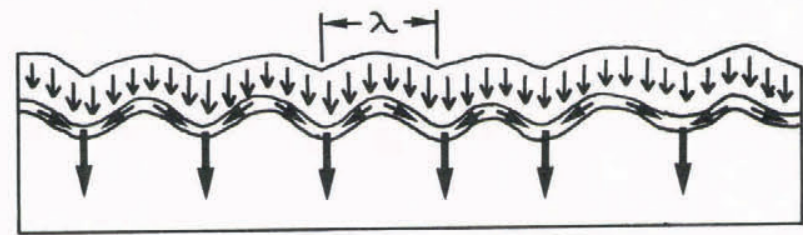

HORIZONTAL DISTANCE

Fig.1. The occurrence of dimple pattern and water movement in a snowcover.

to appear on a snow surface, a saturated layer exists in snow (Nohguchi 1984). Moreover, it is always folded with a constant wavelength determined simultaneously with the formation of the saturated layer. The occurrence of this folding is often followed by the formation of vertical drainage channels due to concentration of water; as a result, the dimple pattern becomes clearer. Folding with a characteristic wavelength suggests that the elastic instability is the trigger forming the regular pattern. Not only elastic deformation but also viscous deformation and solar radiation can explain the growth of dimples. However, viscous deformation and solar radiation cannot explain the wavelength because they are positive feedback mechanisms for any wavelength.

The purposes of this paper are to show the elastic instability of a snowcover with a saturated layer and to present an expression for the wavelength.

\section{THEORY}

Snow is a visco-elastic material, though for quick processes it can be dealt with as an elastic material. To show the instability, consider a homogeneous snow layer (thickness: L, density: $\rho$ ) neighboring a saturated layer at the upper boundary $(\mathrm{z}=\mathrm{L})$ and with a rigid body at the lower boundary $(z=0)$ (Figure 2). To make the theory simple we neglect the horizontal components of elastic displacement because they have little effect on this phenomenon. Then the elastic state of the snow layer can be approximately described by the following equations

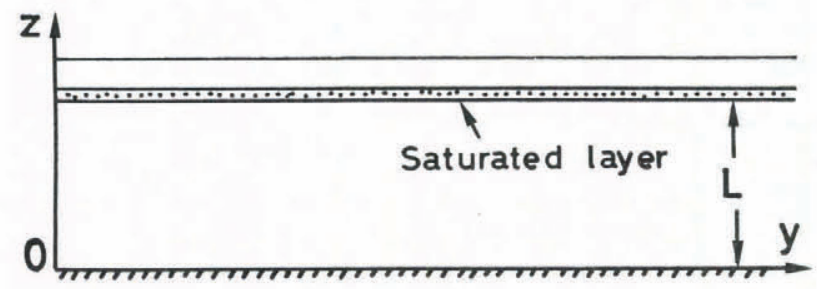

Fig.2. Snowcover with a saturated layer, and coordinate system.

$$
\begin{aligned}
& \partial \sigma / \partial \mathrm{z}+\partial \tau / \partial \mathrm{y}=\rho \mathrm{g} \\
& \sigma=\mathrm{E}(\partial \mathrm{u} / \partial \mathrm{z}), \quad \tau=\mathrm{E}_{\mathrm{s}}(\partial \mathrm{u} / \partial \mathrm{y})
\end{aligned}
$$

where $u$ is the $z$ component of elastic displacement, and $E$ and $E_{S}$ are coefficients of elasticity.

As long as the boundary conditions are independent of $y$, each of $u, \sigma$ and $\tau$ is also independent of $y$. Let the deviations of $u, \sigma$ and $\tau$ from this uniform state be $u^{\prime}, \sigma^{\prime}$ and $\tau^{\prime}$ respectively, then Equation 1 can be rewritten in terms of the deviations as

$$
\begin{aligned}
& \partial \sigma^{\prime} / \partial z+\partial \tau^{\prime} / \partial y=0 \\
& \sigma^{\prime}=E\left(\partial u^{\prime} / \partial z\right), \tau^{\prime}=E_{S}\left(\partial u^{\prime} / \partial y\right)
\end{aligned}
$$

The stability of the uniform state can be examined by giving an infinitesimal deviation (amplitude: 8 , wavenumber: k) of displacement at the upper boundary; the boundary conditions are as follows:

$$
\begin{array}{ll}
u^{\prime}=\delta \sin k y & (\mathrm{z}=\mathrm{L}) \\
\mathrm{u}^{\prime}=0 & (\mathrm{z}=0)
\end{array}
$$

because any deviation can be expressed by superposing it. Let the deviation of normal stress at $z=L$ calculated from Equations 2 and 3 be $\sigma_{\mathrm{e}}{ }^{\prime}$, then we find

$$
\sigma_{e^{\prime}}=\delta \mathrm{k} \sqrt{\mathrm{EE}_{\mathrm{s}}} \operatorname{coth}\left(\sqrt{\mathrm{E}_{\mathrm{s}} / \mathrm{E}} \mathrm{kL}\right) \sin \mathrm{ky}
$$

On the other hand, this displacement deviation at the upper boundary brings about a non-uniform distribution of water in the saturated layer because the water moves to lower position along the upper boundary as mentioned by Wakahama (1963). As a result, it can be reasonably assumed that the deviation $\left(\sigma_{\mathrm{w}}{ }^{\prime}\right)$ of normal stress due to water movement is an increasing function of the displacement deviation $\left(u^{\prime} I_{z=L}\right)$ and vanishes at $u^{\prime} I_{z=L}=0$. Therefore, when the displacement deviation is sufficiently small, $\sigma_{w}$ ' can be first approximately represented as

$$
\sigma_{\mathrm{w}}{ }^{\prime}=\alpha \mathrm{u}^{\prime} \mathrm{I}_{\mathrm{z}=\mathrm{L}}
$$


where $\alpha$ is proportional constant defined by Equation 5 itself. Thus from Equation 3

$$
\begin{aligned}
& \sigma_{w^{\prime}}=\delta \propto g \sin k y \\
& \text { If } \\
& \left|\sigma_{e^{\prime}}\right|<\left|\sigma_{w}{ }^{\prime}\right|
\end{aligned}
$$

this initial deviation as a disturbance becomes larger. Therefore, the uniform state is unstable; the pattern of the wavenumber $k$ (wavelength $\lambda=2 \pi / k$ ) is formed. On the other hand, if not, it is stable; the pattern is not formed.

\section{RESULTS}

From Equations 4, 6 and 7 we can obtain the condition for the occurrence of the instability with the wavenumber $\mathrm{k}$

$$
\begin{aligned}
& (\mathrm{E} / \alpha g \mathrm{~L}) \mathrm{k}^{*}<\tanh \mathrm{k}^{*} \\
& \mathrm{k}^{*}=\sqrt{\mathrm{E}_{\mathrm{s}} / \mathrm{E}} \mathrm{Lk}
\end{aligned}
$$

This indicates that if $\mathrm{E} / \mathrm{\alpha gL}<1$, the uniform state is unstable for a wavenumber less than $k_{c}$, which is the solution of the following equation

$$
(E / \operatorname{ag} L) k^{*}=\tanh k^{*}
$$

and that if $\mathrm{E} / \operatorname{agL}>1$, it is stable for any wavenumber $\mathrm{k}$ as shown in Figures 3 and 4. In general, a natural snowcover can be assumed to be disturbed in every wavenumber. Therefore, we can find that the occurrence of the instability (that is, the formation of the pattern) is governed by the nondimentional parameter $\mathrm{E} / \alpha \mathrm{gL}$ as follows

$$
\begin{aligned}
& \mathrm{E} / \operatorname{\alpha gL}>1: \text { not occur } \\
& \mathrm{E} / \operatorname{\alpha gL}<1: \text { occur }
\end{aligned}
$$

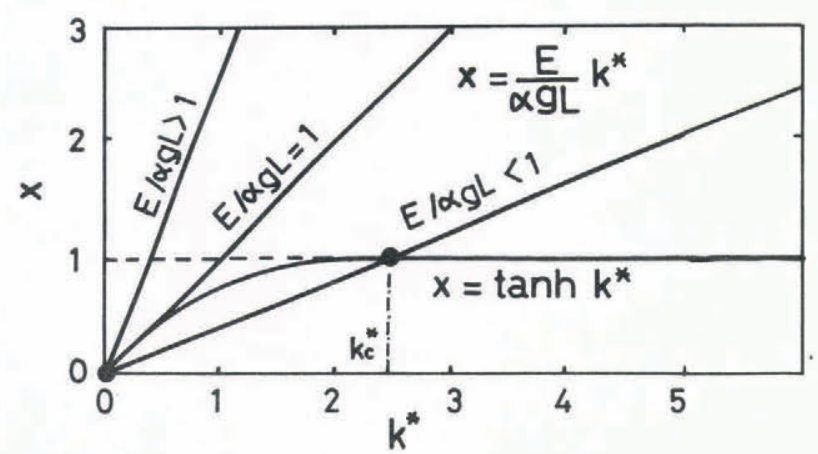

Fig.3. If $E / \alpha g L<1,(E / \alpha g L) k^{*}<\tanh k^{*}$ for $k^{*}<k_{c}^{*}$. Whereas, if $E / \alpha g L>1,(E / \alpha g L) k^{*}>\tanh k^{*}$ for any $\mathrm{k}^{*}$.

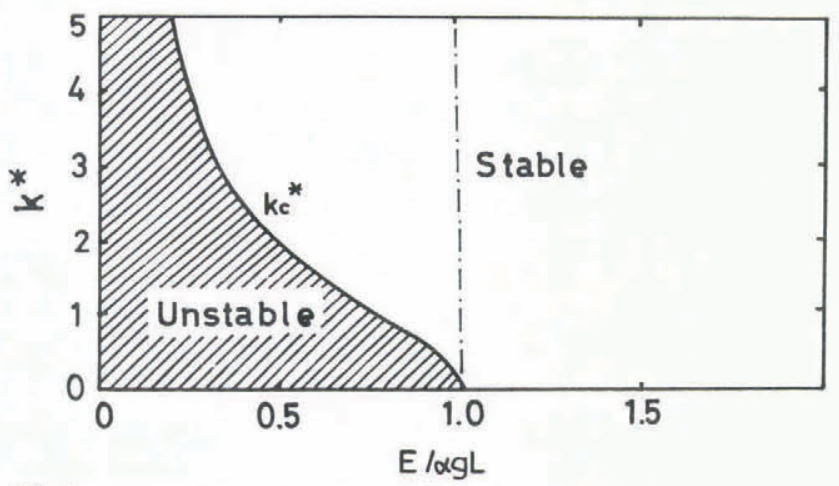

Fig.4. The instability domain for the nondimensional parameter $\mathrm{E} / \mathrm{agL}$ and the nondimentional wave number
When $\mathrm{E} / \mathrm{agL}<1$, the wavelength of the instability is characterized by the minimum wavelength $\lambda_{c}=2 \pi / k_{c}$. From Equation 9 it can be shown that $\lambda_{c}$ increases with increasing $\mathrm{E}$ and $\mathrm{E}_{\mathrm{S}}$. In particular, if $\mathrm{E} / \mathrm{\alpha gL} \ll 1, \lambda_{\mathrm{C}}$ can be represented as

$$
\lambda_{c}=2 \pi \sqrt{E E_{s}} / \alpha g
$$

independent of the thickness $\mathrm{L}$.

\section{COMPARISON WITH OBSERVATIONS}

According to the theory, both the occurrence of new pattern and its wavelength on a new snowcover are determined only by the snowcover at the time when the saturated layer was formed. Therefore, observations to compare with the theory must be carried out simultaneously with the beginning of the occurrence of dimples.

In general, both $\mathrm{E}$ and $\mathrm{E}_{\mathrm{s}}$ increase with increasing snow density (Kojima 1954, and Shinojima 1966). Therefore from Equation 11, $\lambda_{c}$ also increases with increasing snow density $p$. This can be assured by the field observations in Niigata Prefecture, Japan, as shown in Figure 5; it is found that the number of dimples per square meter (f) decreases with increasing snow density directly under the saturated layer. The broken line in Figure 5 represents the following relation of $\mathrm{f}$ to $\rho\left(\mathrm{kg} / \mathrm{m}^{3}\right)$

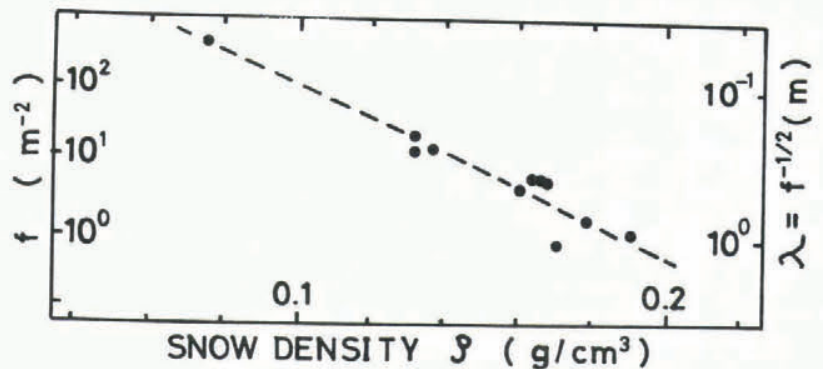

Fig.5. The number (f) of dimples per meter ${ }^{2}$ vs snow density ( $p$ ) just under the saturated layer: in 1983 and 1984 winters, Niigata Prefecture.

$$
f=1 / \lambda^{2} \propto \exp (-0.0534 \rho)
$$

Then, by using Equations 11 and 12, the relation of $\mathrm{E}$ (or $\mathrm{E}_{\mathrm{S}}$ ) to $\mathrm{p}$ can be derived as

$$
E\left(\text { or } E_{S}\right) \propto \exp (0.0267 p)
$$

The nondimentional parameter $\mathrm{E} / \propto \mathrm{gL}$ increases with time and becomes larger than unity, because both $\mathrm{E}$ and $1 / \mathrm{L}$ increase with time owing to snow settlement. Therefore, if the snow layer is sufficiently old, the dimple pattern cannot appear, even if a saturated layer is formed. This theoretical result also is consistent with the fact that the pattern has never occurred for high density $(\rho>200$ $\mathrm{kg} / \mathrm{m}^{3}$ ) as shown in Figure 5 .

As mentioned before, measurements of the snow density must be carried out immediately after the occurrence of dimples, or at latest within several hours. In addition, it must be carried out at the most soft snow just under the saturated layer and before melt-freeze metamorphism. Otherwise, higher density than the right one would be always measured owing to snow settlement and melt-freeze metamorphism.

\section{CONCLUSION}

It was theoretically shown that the uniform state of a snowcover with a saturated layer is unstable when the nondimentional parameter $\mathrm{E} / \mathrm{\alpha gL}<1$. Then the wavelength of the instability is characterized by the minimum wavelength $\lambda_{\mathrm{c}}$ : In particular, when $\mathrm{E} / \propto \mathrm{gL} \ll 1, \lambda_{\mathrm{c}}$ increases with increasing snow density and is independent of the thickness of the snow layer. These results are assured by the field observations. Thus we conclude that the elastic instability of a snowcover with a saturated layer triggers the occurrence of the dimple pattern. 


\section{REFERENCES}

Gerdel R W 1954 The transmission of water through snow. Transactions of the American Geophysical Union 35: 475-485

Kojima K 1954 Visco-elastic property of snow. Low Temperature Science A, 12: 1-13

Nohguchi Y 1984 [Formation of dimple pattern in snow I.] Report of the National Research Center for Disaster Prevention 33: 237-54 (in Japanese)

Seligman G 1936 Snow structure and ski fields. Brussels, Jos. Adam

Shinojima K 1966 Visco-elastic deformation of snow. In Oura $\mathrm{H}$ (ed) Physics of Snow and Ice. Sapporo, Institute of Low Temperature Science : 875-907

Wakahama G 1963 The infiltration of melt water into snow cover I. Low Temperature Science A, 21: 45-75

Wankiewicz A, de Vries J 1978 An inexpensive tensiometer for snow-melt research. Journal of Glaciology 20: $577-584$ 